\title{
The Early Evolution of Dense Stellar Systems
}

\author{
C. J. Clarke \\ Institute of Astronomy, Madingley Road, Cambridge, UK \\ email: cclarke@ast.cam.ac.uk
}

\begin{abstract}
The early evolution of dense stellar systems is dominated by the majority mass component - the gas - and so any credible modeling of the first Myr or so of a cluster's life inevitably involves hydrodynamical simulations. Such simulations have increased considerably in sophistication over the last few years and are now beginning to incorporate the effects of stellar feedback, thus enabling one, for the first time, to model the formation of populous clusters. In this review I focus on two issues that have arisen from the simulations - the relationship between maximum stellar mass and cluster mass, and the issue of the maximum density that is attainable during the cluster formation process. I also report on the first results of new simulations that model feedback from ionising radiation.
\end{abstract}

Keywords. hydrodynamical simulations, stars: formation, ISM: evolution

\section{Introduction}

Most of those attending this Symposium will not have considered in any detail the early (hydrodynamic) stages of cluster formation. Indeed, many would probably consider it to be too inherently messy to be appealing, in contrast to the relatively well posed problems concerning a cluster's further evolution as an Nbody system.

There are in fact two potentially valid reasons to feel this way about hydrodynamic simulations of cluster formation. The first is the issue of numerical accuracy; in dissipative systems, one cannot rely on simple constraints such as total energy, and even where codes can readily reproduce simple one dimensional tests, there remain some doubts about their ability to model the sort of complex three dimensional flows encountered in turbulent star forming regions. In this situation, an important check is provided by comparisons between different numerical codes and it is particularly encouraging that, whereas Lagrangian codes (i.e. SPH) have blazed the trail in this area, there is the prospect that in future it will become possible to undertake detailed comparisons with Eulerian (Adaptive Mesh Refinement) codes.

The second reason for considering this evolutionary phase as 'messy' is however intrinsic to the problem, and derives from the fear that - even in the case of perfect numerical accuracy - the evolution may be unduly sensitive to details of the initial gas distribution. This is a particular issue once one considers the effects of feedback from massive stars, given the extreme density sensitivity of the photoionisation process. One then has to worry that even the gross outcome of a simulation (for example, whether a proto-cluster remains bound or not), could depend on a detailed knowledge of the gas filling factor and morphology, which could never be known in practice. It is however still unclear (given the small number of simulations that have explored this issue) whether such simulations can be more than exercises in detailed 'weather forecasting' and how easy it will be to derive general trends and insights from them. 

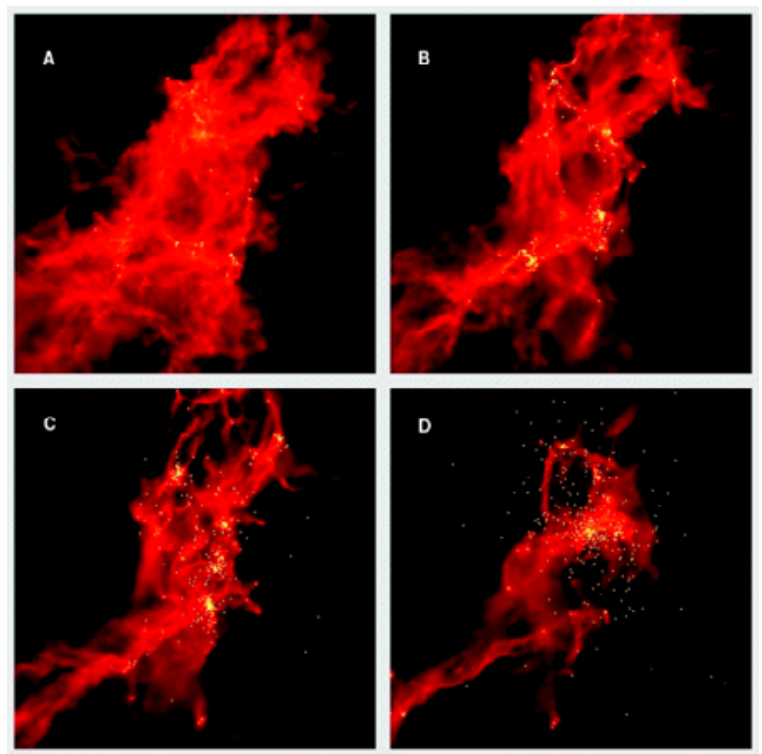

Figure 1. The hierarchical assembly of a star cluster from turbulent fragmentation simulations of Bonnell et al. 2003.

Notwithstanding these two caveats, it is evidently of great interest to all those working on the evolution of dense stellar systems to understand what factors control such properties as the stellar mass spectrum and its dependence on environment, as well as considering the conditions of possibly extreme stellar density that may prevail at early times. In this contribution, I paint a thumbnail sketch of the expectations of turbulent fragmentation simulations and show that a key insight of recent years, both observationally and theoretically, is that clusters are assembled in a hierarchical (bottom-up) manner. I then relate this picture to the observed relationship between maximum stellar mass and cluster mass. Next I turn to the question of the maximum density achieved in young star clusters, and show that the maximum density - following adiabatic accretion of gas onto a cluster core - exceeds the mean cluster density by a factor depending on the square of the cluster mass. This result then implies that the runaway collisional growth of stars in an adiabatic core is limited to the case of more massive clusters. Finally, I review the results of pilot simulations that include feedback from massive stars and show that it is currently unclear whether the net effect of feedback on star formation is positive or negative. I also highlight the difficulty in using observational diagnostics to identify examples of triggered star formation.

\section{The hierarchical assembly of stellar clusters in turbulent molecular clouds}

Fig. 1 contains several frames from the (SPH) simulation of Bonnell, Bate and Vine 2003, which depict the hierarchical assembly of a star cluster from a turbulent cloud. In this simulation, the (piecewise polytropic) gas is subject to an initial turbulent velocity field, whose spectral slope is set so as to replicate the observed size-line width relation in molecular clouds (Larson 1981). These motions are supersonic (Mach $\sim 10$ ) on the cloud scale $(\sim 1 \mathrm{pc})$ and lead to the development of a filamentary network of shocks on the cloud crossing timescale $\left(\sim 2 \times 10^{5}\right.$ years $)$. Since the energy dissipated in shocks 


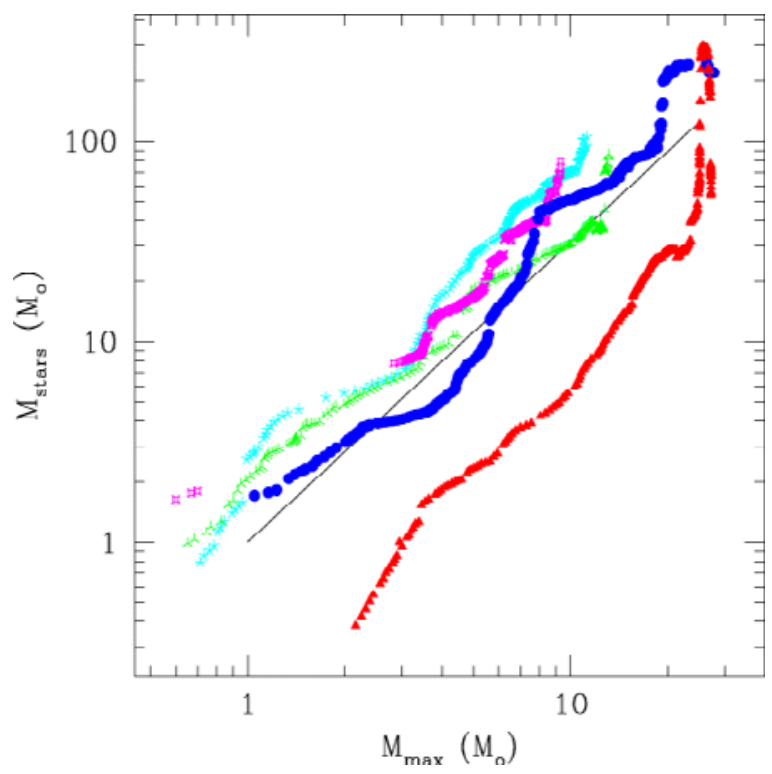

Figure 2. The assembly history of massive stars: the mass of the most massive star in its cluster is plotted as a function of the total mass in stars within $0.1 \mathrm{pc}$. From

Bonnell et al. 2004.

is not replenished in these simulations, the subsequent evolution involves not only the fragmentation of over-dense (shocked) regions but also the over-all collapse of the cloud. Thus, whereas, star formation proceeds initially in a small $\mathrm{N}$ cluster mode in regions of the highest density (where the Jeans mass is lowest), these clusters subsequently merge and form successively larger structures. Such a picture of hierarchical cluster formation is consistent with the wealth of small scale structure seen in star forming regions (Guillout et al. 1998, Gouliermis et al. 2000, Elmegreen \& Elmegreen 2001).

A striking property of such simulations is that in each cluster, the most massive star is generally located close to the cluster centre (where it intercepts the densest accretion flow and hence grows most rapidly). Following cluster mergers, the most massive star sinks rapidly to the gas-rich core of the new cluster and grows further in mass. Thus, in the simulations, the mass of the most massive star increases with the mass of the parent cluster, although less than linearly: Fig. 2 depicts the growth in mass of several stars in the simulations of Bonnell et al. 2004, each of which are the most massive members of their cluster, plotted as a function of the mass of the parent cluster (as represented by the mass contained within $0.1 \mathrm{pc}$ of the most massive star). Although both quantities grow in time, as the most massive star and its neighbours accrete gas, there are phases when new members arrive in the cluster core (near vertical sections in Fig. 2) which flattens the slope of the $M_{\max }$ versus $M_{\text {clus }}$ relation to $M_{\max } \propto M_{\text {clus }}^{2 / 3}$.

It is notable that this slope is close to the relationship $M_{\max } \propto M_{\text {clus }}^{1 / 1.35}$ which is expected by random drawing from a Salpeter IMF. In fact, it is found that the IMF within the clusters is indeed close to a Salpeter distribution and that the most massive star is close to that expected from populating this distribution with a finite number of stars appropriate to the cluster mass.

The observational situation is depicted in Fig. 3 - an increase in maximum stellar mass as the cluster scale increases (up to a cluster mass of a few $\times 10^{4} M_{\odot}$ - i.e. $\sim 100 \mathrm{OB}$ stars) and then a flattening off with a maximum recorded value of around $150-200 M_{\odot}$. 


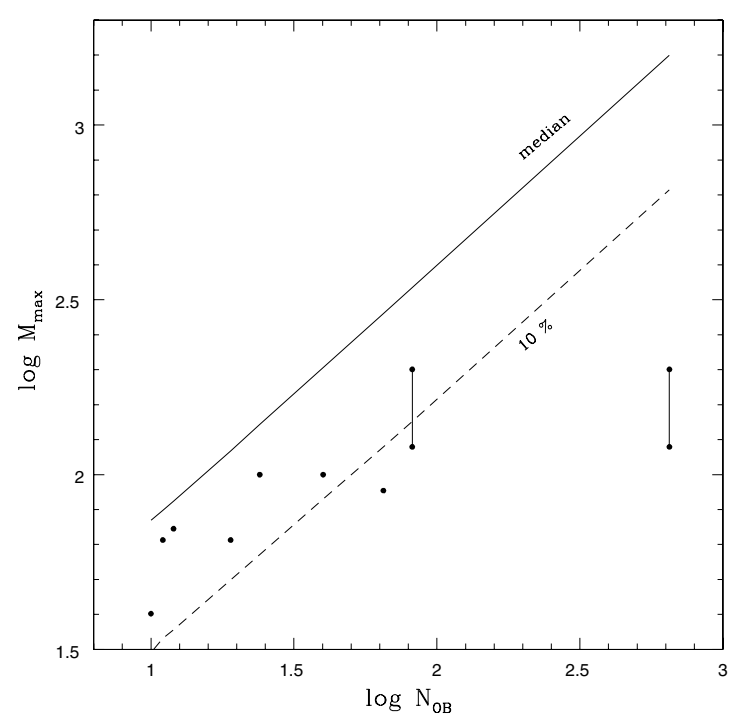

Figure 3. The observed maximum stellar mass of clusters plotted as a function of the cluster richness (number of OB stars). Also shown is the median and tenth percentile of the expected distribution if stars are drawn at random from an untruncated Salpeter IMF. Based on Oey \& Clarke 2005.

The slope of the relationship at lower masses is consistent with random drawing from a Salpeter IMF (although the values are somewhat low: the fact that the points all lie below the expected median relation is however not statistically significant). If one interprets this relationship as being purely a consequence of the statistics of random drawing then, although the median for a large sample of clusters would be expected to lie along the line shown, there would be considerable scatter in both directions and there would be nothing physical that would rule out the occasional discovery of a low mass cluster containing a very massive star.

Whereas it is debatable whether the observed $M_{\text {max }}, M_{\text {clus }}$ relation is statistical or physical at lower masses, it is clear that at higher masses, the apparent saturation of $M_{\text {max }}$ is physical. Indeed, the probability that in a cluster on the scale of R136 (containing $\sim 600 \mathrm{OB}$ stars) the most massive star should be as low as is observed would be $10^{-15}$ according to a random drawing model (Oey \& Clarke 2005). It is however unclear what physics should operate at this mass scale so as to preclude further mass growth (for example, a feedback mechanism that is often invoked in relation to the assembly of massive stars - radiation pressure on dust - becomes effective at the $\sim 10 M_{\odot}$ scale, where the IMF is in fact featureless). Alternatively, the upper mass limit may not relate to star formation but to subsequent mass loss (see, e.g. Smith \& Owocki 2006, Belkus et al. 2007).

Since the simulations (which in this case incorporate no feedback) can so well reproduce the observed $M_{\max }, M_{\text {clus }}$ relationship at low masses, it is instructive to enquire whether this effect should be regarded as statistical or physical in the simulations. This essentially boils down to examining the scatter in the histories of star and cluster growth in the $M_{\max }, M_{\text {clus }}$ plane. In as far as the limited number of accretion histories contained in Fig. 2 can provide any insight into the problem, it would appear that the relationship in the simulations is actually statistical. Although the tracks follow an over-all trend, they do exhibit some scatter (for example, the right hand trajectory exhibits a much larger 
value of $M_{\max }$ at given $M_{\text {clus }}$ than the others, presumably reflecting some stochastic variation in the accretion history of its natal cluster). This means that there are not factors which physically prevent the acquisition of a higher $M_{\max }$ value at low $M_{c l u s}$, but just that such an outcome is relatively rare. Pursuing this line of thought, we see that if we were to 'dry merge' a number of clusters (i.e. combine their stellar components without changing their stellar masses), the resulting merged cluster would still have a higher maximum stellar mass than the majority of the constituent clusters from which it formed. This would suggest that we cannot appeal to the onset of 'dry mergers' in order to explain the observed saturation of $M_{\max }$ at $150-200 M_{\odot}$. We however stress that such a conclusion is necessarily preliminary, given the small number of accretion histories analysed to date.

\section{The maximum stellar density and the possibility of stellar collisions}

Examination of the simulations reveals that clusters form at the intersections of filamentary shocks which channel an accretion flow onto the clusters. The timescale for mass doubling due to this flow is set by the free fall timescale of the large scale structure (parent cloud) which is generally longer than that of the cluster itself. Thus the cluster acquires mass in the adiabatic regime and it is an easy matter to demonstrate that the twin requirements of virial equilibrium and the preservation of the adiabatic invariant $\Sigma p_{i} . r_{i}$, (where $p_{i}$ and $r_{i}$ are respectively stellar positions and momenta), imply that the cluster radius shrinks as the inverse cube of the cluster mass (i.e. $R \propto M^{-3}$ ). $\dagger$ This adiabatic mass-radius relationship implies an extremely steep dependence of cluster density on mass $\left(\rho \propto M^{10}\right)$; thus since the cluster free fall timescale declines steeply (as $M^{-5}$ ), any cluster that enters the adiabatic regime will become increasingly adiabatic as time goes on. Thus Bonnell, Bate \& Zinnecker (1998) argued that the stellar density would eventually rise to the point where physical collisions between stars would become important. Given the possible problems that have been discussed about the viability of forming the most massive stars by accretion (due to the effect of radiation pressure on dust: see e.g. Wolfire and Cassinelli 1987, Edgar \& Clarke 2004) this appeared to be an attractive alternative mechanism for massive star formation.

A simple estimate of collisional cross sections for single $\mathrm{O}$ stars leads to the benchmark

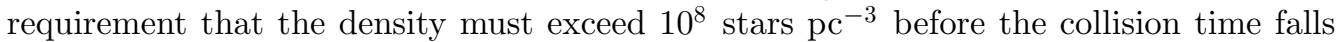
below $10^{5}$ years. This exceeds the densities of the densest observed star clusters by two orders of magnitude and therefore - if such conditions are ever met - they must belong to a deeply embedded, and observationally inaccessible, phase of cluster formation. We note in passing that Moraux, Lawson and Clarke (2007) have recently inferred initial densities of $\sim 10^{8} \mathrm{pc}^{-3}$ for the Eta Cha association, based on the assumption that the observed lack of brown dwarfs and wide binaries in this cluster is a result of dynamical effects. (It should be stressed however that Eta Cha does not contain stars that are sufficiently large in cross section to have collided even at such high densities.)

The above density estimates make the (pessimistic) assumption of (gravitationally focused) encounters between single stars. (Note that the velocity dispersion rises rather gently with density in the adiabatic phase $\left(v \propto \rho^{1 / 5}\right)$ and so gravitational focusing can remain important even at high densities. By the same token, velocities can remain less than the stellar escape velocity and thus collisions can lead to mergers rather than

$\dagger$ Note that this argument is equally applicable if, instead of a cluster and parent cloud, we instead consider a dense cluster core gaining mass via inflow from a gas-rich outer cluster. 
disruptions). Bonnell \& Bate (2002) however found that the main route to stellar collisions was via the gravitational perturbation of close binaries. In practice, collisions are likely in the case of binaries whose separations are around $\sim 10$ stellar radii (i.e. around an A.U. or so in the case of massive stars). Thus, if the cluster contains a significant population of primordial binaries on the scale of 1 A.U., the densities required for collisions are reduced by around an order of magnitude.

The above description suggests that adiabatic accretion leads to an inexorable rise in core density and that therefore - given a continuing supply of accretable material - the eventual realisation of conditions conducive to stellar collisions in the core is inevitable. Nevertheless, Bonnell \& Bate (2002) found instead that, in the case of their simulation of a cluster containing $\sim 10^{3}$ stars, the maximum core density saturated at around 5 orders of magnitude times its initial value. At this point, the shrinkage due to adiabatic accretion was offset by puffing up of the cluster core by two body effects (specifically the injection into stellar motions of kinetic energy released by the creation or hardening of binaries). The maximum density attained was not quite sufficient (assuming a cluster with average density comparable to the Orion Nebula Cluster) to give rise to collisions unless (like Bonnell \& Bate 2002) one applies artificially enhanced collision cross sections.

Recently, Clarke \& Bonnell (2007) have developed this insight in order to determine the properties of host systems whose cores should be able to attain the densities necessary for stellar collisions. The maximum core density is obtained at the point that the timescale for core shrinkage (which is the mass doubling timescale in the core, $t_{\dot{M}}$ ) becomes longer than the timescale for puffing up by two body processes, a timescale that can (in the case of a small core, containing $N_{c}<100$ stars) be approximated as $\sim N_{c}$ core crossing times (Bonnell \& Clarke 1999). $t_{\dot{M}}$ is simply the ratio of the core mass to the mass inflow rate, $\dot{M}$, where $\dot{M}$ is given (in the case of the free fall collapse of a gaseous reservoir of mass $M_{g}$ ) by $M_{g} / t_{d y n}$, with $t_{d y n}$ being the dynamical timescale at the cluster half mass radius. Putting all this together, one obtains the very simple result:

$$
\rho_{\max }=\bar{\rho}\left(\frac{M_{g}}{\bar{m}_{c}}\right)^{2}
$$

where $\bar{m}_{c}$ is the mean stellar mass in the core. Substituting $M_{g} \sim 1000 M_{\odot}$ and $\bar{m}_{c} \sim 3 M_{\odot}$ one recovers the result (obtained numerically by Bonnell \& Bate 2002) that the core density growth should saturate at around five orders of magnitude above the mean value. One may then compute the mean number of collisions per star, $f_{\text {coll }}$, as being the product of the collision rate at this maximum density and the core shrinkage timescale. After a little manipulation, we obtain:

$$
f_{\text {coll }}=N_{c}\left(\frac{v}{v_{*}}\right)^{2}
$$

where $v$ and $v_{*}$ are respectively the cluster velocity dispersion and escape velocity from the stellar surface. Given that $v$ is typically a few $\mathrm{km} \mathrm{s}^{-1}$ and $v_{*}$ is several hundreds of $\mathrm{km} \mathrm{s}^{-1}$, it follows that stellar collisions are likely to be important only in the case of cluster cores containing $>10^{4}$ stars.

The above estimate is necessarily a rough one and brushes aside a number of issues such as the feasibility of continued accretion in the face of stellar feedback, as well as the effects of mass segregation within a dynamically decoupled (adiabatic) core. It also, by focusing on the fate of a core containing $N_{c}$ stars, does not make any predictions about the relationship between $N_{c}$ and the number of stars in the parent cluster $\left(N_{*}\right)$, apart from the obvious restriction that $N_{c}<N_{*}$. It nevertheless suggests that runaway stellar collisions should be a possible outcome only in the case of clusters with $N_{*}>>10^{4}$. 
A preliminary conclusion, therefore, is that one should not expect runaway stellar collisions in the sorts of environments in which they have been discussed hitherto (i.e. the Orion Nebula Cluster, the Arches Cluster or R136 in 30 Doradus). This is consistent with the fact that even in these most populous clusters, there is no evidence for the presence of stars with masses in excess of $150-200 M_{\odot}$ (Figer et al. 1998), although it may be argued that runaway collisions may occur in these regions in the future through the purely stellar dynamical operation of the mass segregation (Spitzer) instability (Spitzer 1969, Gürkan et al. 2004). On the other hand, more populous systems, such as globular clusters or super star clusters (SSCs) are certainly candidate systems for accretion induced runaway stellar collisions, the main requirement being a strong radial inflow of gas into a populous core region. This result has obvious ramifications for the production of intermediate mass black holes (IMBHs) in globular clusters. $\dagger$

\section{The role of feedback}

The vast majority of cluster star formation simulations performed to date omit the effects of feedback, whether on the scale of accretion onto individual stars (where radiation pressure on dust may be significant) or on a cluster wide scale, due to either thermal ionisation or the mechanical feedback supplied by stellar winds. Simulations involving feedback are in their infancy and have thus far served more to patent the viability of the algorithms rather than permitting any exploration of parameter space. Nevertheless, current work does allow one to draw a few preliminary conclusions and cautionary notes.

In the case of feedback from the ionising radiation from massive stars, Dale and collaborators have recently succeeded in implementing this effect in SPH simulations (see Dale et al. (2007) for a demonstration that, in the dense environments of molecular cloud cores, the simple - on the spot - assumption employed by the algorithm is validated by comparison with Monte Carlo radiative transfer calculations). To date, pilot simulations have applied such feedback to the case of an internal ionising source in the core of a dense, highly inhomogeneous protocluster cloud (Dale et al. 2005) and also to the case of an external ionising source irradiating an initially unbound cloud region (Dale et al. 2006). In the former case (see Fig. 4), the source ionises and drives a set of thermally driven outflows via low density channels, while at the same time, the lateral expansion of such ionised channels compresses intervening filaments of dense, inflowing gas. The result is a complex combination of positive feedback (induced star formation in the compressed dense filaments) and negative feedback (inhibition of inflow into into the cluster core, due to entrainment of material in outflows), such that current simulations do not have the resolution to determine the sign of the net effect. In the case of external irradiation of an unbound cloud, the net effect is certainly positive, inasmuch as gas which in the absence of feedback, would have just expanded away from the cloud core is instead impacted by the ionisation front and returned to the cloud core, shocking and producing additional stars in the process. A notable outcome of this simulation is that it is apparently rather hard to distinguish observationally between those stars whose formation is triggered in this way, and those that arise spontaneously in the absence of feedback. Comparison between the feedback simulations and control experiments suggest that there is no kinematic signature of triggering (Dale et al. 2006): although the free expansion speed of ionised gas is around $10 \mathrm{~km} \mathrm{~s}^{-1}$, this is decreased, by mass loading,

$\dagger$ Note that if stellar collisions are ever important - either as a result of adiabatic accretion or the action of the mass segregation instability - it has still to be demonstrated that the collision product could grow into the very massive (hundreds of solar masses or more) regime instead of undergoing avoid violent mass loss: see e.g. Belkus et al. (2007). 


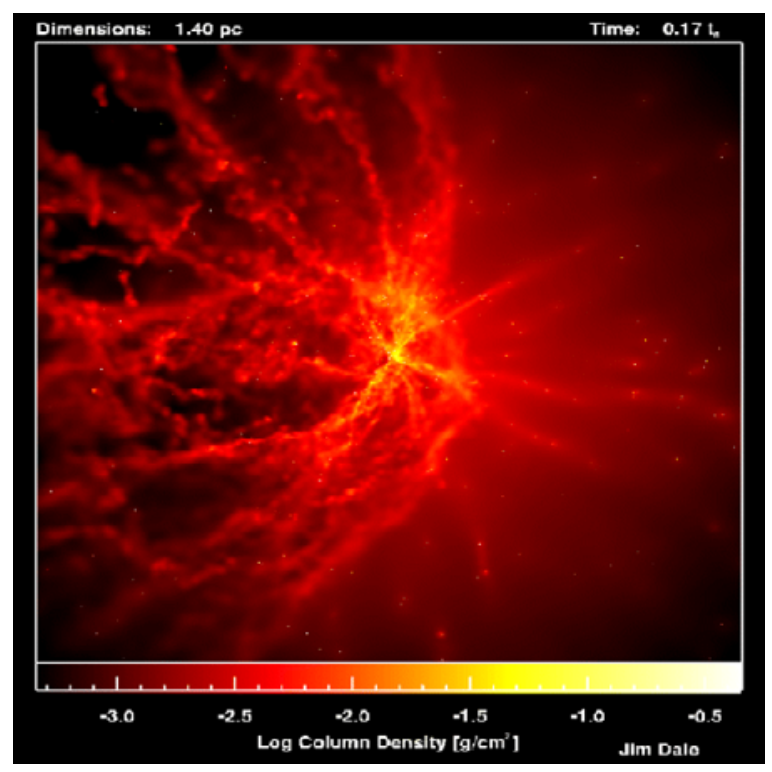

Figure 4. Simulation of the formation of a star cluster including the feedback of ionising radiation from massive stars. Dale et al. 2005.

to a few $\mathrm{km} \mathrm{s}^{-1}$, which is in any case comparable with the typical speed of turbulent motions in molecular clouds.

Perhaps the most notable - and salutary - result to emerge from these pilot simulations is simply that, in a highly inhomogeneous medium, the gas absorbs a quantity of thermal and kinetic energy that far exceeds the cloud's gravitational binding energy, does not imply that the nascent cluster will become gravitationally unbound (Dale et al. 2005). This is simply because most of the energy injected is carried off by material traveling at close to $10 \mathrm{~km} \mathrm{~s}^{-1}$, which is considerably greater than the cluster escape velocity, while the bulk of (dense) gas in the simulation (and stars) remains bound. This effect is in addition to the often noted fact that the quantity of energy absorbed by the gas is itself many orders of magnitude less than the energy output of the ionising source, due to radiative losses in the gas. Taken together, these two effects imply that energetic arguments about the viability of cluster disruption should - if not backed up by simulations - be treated with great caution.

In parallel with these first steps towards modeling ionisation feedback, Dale et al. have recently embarked on SPH simulations which include the mechanical feedback from stellar winds. Since the mechanical luminosity of the stellar wind rises much more steeply with stellar mass than does its ionising luminosity (Vink et al. 2001), it follows that feedback from stellar winds is likely to be of increasing significance in populous clusters which contain the most massive stars. Ultimately, of course, one would wish to combine the effect of photoionisation and mechanical energy injection as in existing galactic scale simulations which assume a smooth density stratification of the interstellar medium (e.g. Tenorio Tagle et al. 1999). A medium term challenge, which must be met if one is to provide any credible insights into cluster formation, is to incorporate these effects into the complex and highly dynamical environments in which star clusters form.

\section{References}

Belkus, H., Van Bever, J., \& Vanbeveren, D. 2007, ApJ, 659, 1576 
Bonnell, I., Bate, M., \& Zinnecker, H. 1998, MNRAS, 298, 93

Bonnell, I. \& Bate, M. 2002, MNRAS, 336, 659

Bonnell, I. A., Bate, M. R., \& Vine, S. G., 2003, MNRAS, 343, 413

Bonnell, I., Vine, S., \& Bate, M. 2004, MNRAS, 349, 735

Bonnell, I. A. \& Clarke, C. J. 1999, MNRAS, 309, 461

Clarke, C. J. \& Bonnell, I. A. MNRAS, submitted

Dale, J., Bonnell, I., Clarke, C., \& Bate, M. 2005, MNRAS, 358, 291

Dale, J., Clark, P. C., \& Bonnell, I., 2006, MNRAS, submitted

Dale, J., Ercolano, B., \& Clarke, C. 2007, MNRAS, in press (arXiv:0705.3396)

Edgar, R. G. \& Clarke, C. J. 2004, MNRAS, 349, 678

Elmegreen, B. G. \& Elmegreen, D. M. 2001, AJ, 121, 1507

Figer, D. F, Najarro, F., Morris, M., McLean, I. S., Geballe, T. R., Ghez, A. M., \& Langer, N. 1998, ApJ, 506, 384

Gouliermis, D., Kontizas, M., Korakitis, R., Morgan, D. H., Kontizas, E., \& Dapergolas, A. 2000, AJ, 119, 1757

Guillout, P., Sterzik, M. F., Schmitt, J. H. M. M., Motch, C., \& Neuhaeuser, R. 1998, A \& A, 337,113

Gürkan, M. A., Freitag, M., \& Rasio, F. 2004, ApJ, 604, 632

Larson, R. B. 1981, MNRAS, 194, 809

Moraux, E., Lawson, W. \& Clarke, C. J. 2007, A\&A, 473, 163

Oey, S. M. \& Clarke, C. J. 2005, ApJL, 620, 430

Smith, N. \& Owocki, S. P. 2006, ApJ, 645, L45

Spitzer, L. J. 1969, ApJ, 158, L139

Tenorio-Tagle, G., Silich, S. A., Kunth, D., Terlevich. E., \& Terlevich, R. 1999, MNRAS, 309, 332

Vink, J., de Koter, A., \& Lamers, H. J. G. L. M. 2001, A \& A, 369, 574

Wolfire, M. G. \& Casinelli, J. P. 1987, ApJ, 319, 850 